\title{
Bewölkungskartierung über der Schweiz mit Wettersatellitenbildern und Bodenbeobachtungen
}

\section{Einleitung und Zielsetzung}

Am Geographischen Institut der Universität Bern (GIUB) wurde im Auftrag der eidgenössischen Fachkommission für die Nutzung der Sonnenenergie (KNS) ein Sonnenenergie-Kataster für die Schweiz erstellt (KUNZ et al., 1984). Diese Studie war Anlaß dazu, eine Bewölkungskarte zu erstellen, die sowohl auf der flächenhaften Information von Wettersatelliten basiert als auch die langjährigen Beobachtungsreihen von Bodenstationen einbezieht. Damit sollen die Vorteile beider Beobachtungsmethoden kombiniert und optimal genutzt werden.

\section{Datenmaterial}

Für Bewölkungsuntersuchungen spielen zwei Beobachtungsmethoden eine bedeutende Rolle: Bodenund Satellitenbeobachtungen. Die beiden Methoden unterscheiden sich in wesentlichen Punkten.

\subsection{Terrestrische Bewölkungsbeobachtungen}

Terrestrische Bewölkungsbeobachtungen werden täglich zu den synoptischen Terminen 7 Uhr, 13 Uhr und 19 Uhr MEZ durchgeführt. Auf den meisten Stationen beurteilt der Beobachter die Bewölkungssituation, ohne auf technische Hilfsmittel zählen zu können. Bewölkungsangaben weisen deshalb eine gewisse Subjektivität auf.

Die Bedeckung des Gesichtsfeldes mit Wolken wird gewöhnlich in Achteln geschätzt. Der Beobachter projiziert bei diesem Vorgang die Bewölkung zentralperspektivisch aufs Himmelsgewölbe. Damit sind folgende Konsequenzen verbunden:

1. Wolkenlücken können vor allem bei cumuliformer Bewölkung nicht erkannt werden (siehe Fig. 1, Feld D2). Die Bewölkungsschätzung fällt in diesem Falle zu hoch aus.

2. Der Beobachter bezieht Wolken, die unmittelbar über ihm liegen, in weit stärkerem Maße in die Schätzungen ein als Wolken am Rande des $\mathrm{Ge}$ sichtsfeldes. Obwohl in B1/B2 der Figur 1 die Bewölkung deutlich geringer ist als in $\mathrm{C} 1 / \mathrm{C} 2$, wird in beiden Situationen eine Bedeckung von einem Achtel beobachtet. Damit stehen einander Wolken- felder gegenüber, die im ersten Falle $6,6 \mathrm{~km}^{2}$, im zweiten $4641 \mathrm{~km}^{2}$ bedecken (Wolkenbasis $4 \mathrm{~km}$, Sichtweite $40 \mathrm{~km}$, Horizontüberhöhung $4 \mathrm{~km}$ ). Verändert sich im Wolkenkranz, der das Gesichtsfeld randlich umgibt, die Bewölkung um einige $100 \mathrm{~km}^{2}$, schlägt sich dies in der Schätzung nicht nieder. Anders im zentralen Bereich, wo bei den obigen Voraussetzungen ein Wolkenfeld von $317 \mathrm{~km}^{2}$ bereits mit sieben Achteln registriert wird. Trotz großem Gesichtsfeld von beinahe $5000 \mathrm{~km}^{2}$ (ca. $1 / 8$ der Fläche der Schweiz) spielt deshalb für die Schätzung nur ein kleiner Kreissektor im Bereich der Bodenstation eine Rolle.

Bodenbeobachtungen ergeben nur dort ein flächendeckendes Bild der Bewölkung, wo Stationen nahe beieinander liegen. In Gebieten geringer Stationsdichte ist man zu Interpolationen gezwungen. Diese Lücken im Stationsnetz sind besonders dort schwierig zu schließen, wo die Bewölkung durch den Untergrund (Relief, Vegetation, Temperatur) zahlreichen Modifikationen unterworfen ist - Verhältnisse, wie sie beispielsweise im schweizerischen Alpenraum anzutreffen sind.

Bodenbeobachtungen werden in der Schweiz seit 1846 systematisch durchgeführt (Genf, Großer St. Bernhard). Von 81 Stationen sind Bewölkungswerte der Periode 1931 bis 60 greifbar. Auf der Basis dieses umfangreichen Zahlenmaterials läßt sich eine Bewölkungsklimatologie aufbauen. SCHÜEPP (1978) hat diese Daten als Grundlage für sein Bewölkungskarte verwendet.

\subsection{Bewölkungsbeobachtung mit Wettersatelliten}

Zwei grundsätzlich verschiedene Satellitensysteme dienen zur Klima- bzw. Wolkenbeobachtung aus dem All: Polarumlaufende Satelliten und geostationäre Satelliten (z. B. Meteosat-2). NOAA-6,7 und 8, polarumlaufende Wettersatelliten der Serie TIROS-N, erzeugen Bildstreifen von $2500 \mathrm{~km}$ Breite, in denen jeder Punkt der Erde täglich 4 bis $6 \mathrm{mal}$ enthalten ist. Meteosat-2 produziert Aufnahmen der ganzen Erdscheibe im Abstand von einer halben Stunde.

Martin Heeb, dipl. Geograph, Geographisches Institut der Universität Bern, Hallerstrasse 12, 3012 Bern 


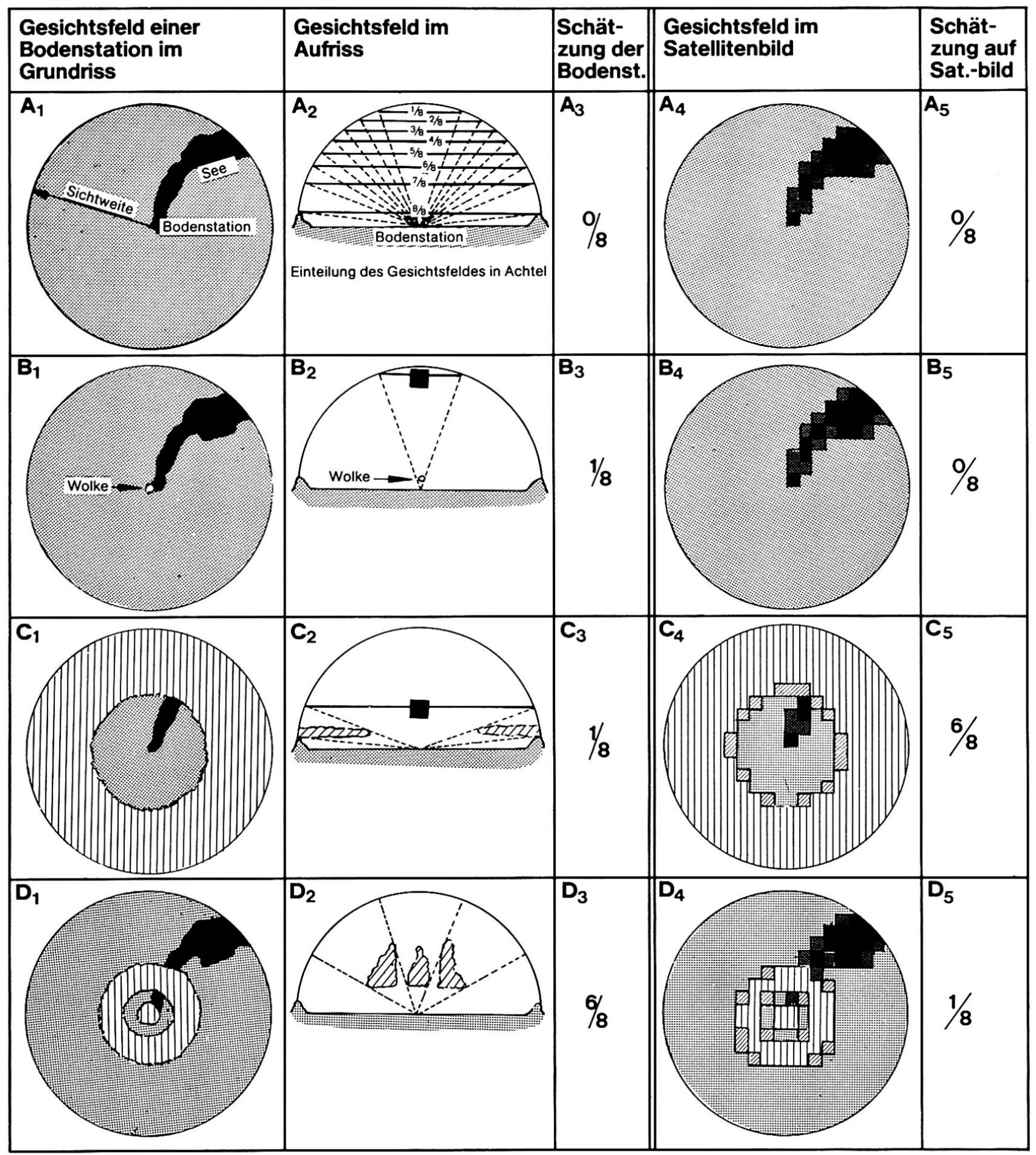

Figur 1 Vergleich von Boden- und Satellitenbeobachtung am Beispiel von hypothetischen Wolkenverteilungen.

Die erwähnten Satelliten verfügen über einen Scanner, der die Erdoberfläche in ein Mosaik von Einzelpunkten (Pixeln) zerlegt, die bei NOAA 6,7 und 8 auf der Subsatellitenlinie eine Seitenlänge von $1,1 \mathrm{~km}$ aufweisen und bei Meteosat-2 in Mitteleuropa $2,5 \times 4 \mathrm{~km}^{2}$ messen. Die Sensoren der Wettersatelliten sind in der Lage, Messungen der Strahlung in ver- schiedenen Spektralbereichen vorzunehmen. Meteosat-2 verfügt über einen Sensor, der die Albedo der kurzwelligen, sichtbaren Strahlung mißt, und über zwei Sensoren (Kanäle), die langwellige Infrarotstrahlung registrieren. NOAA-6, 7 und 8 sind mit vier Infrarotkanälen und einem Kanal im sichtbaren Spektralbereich ausgerüstet. 


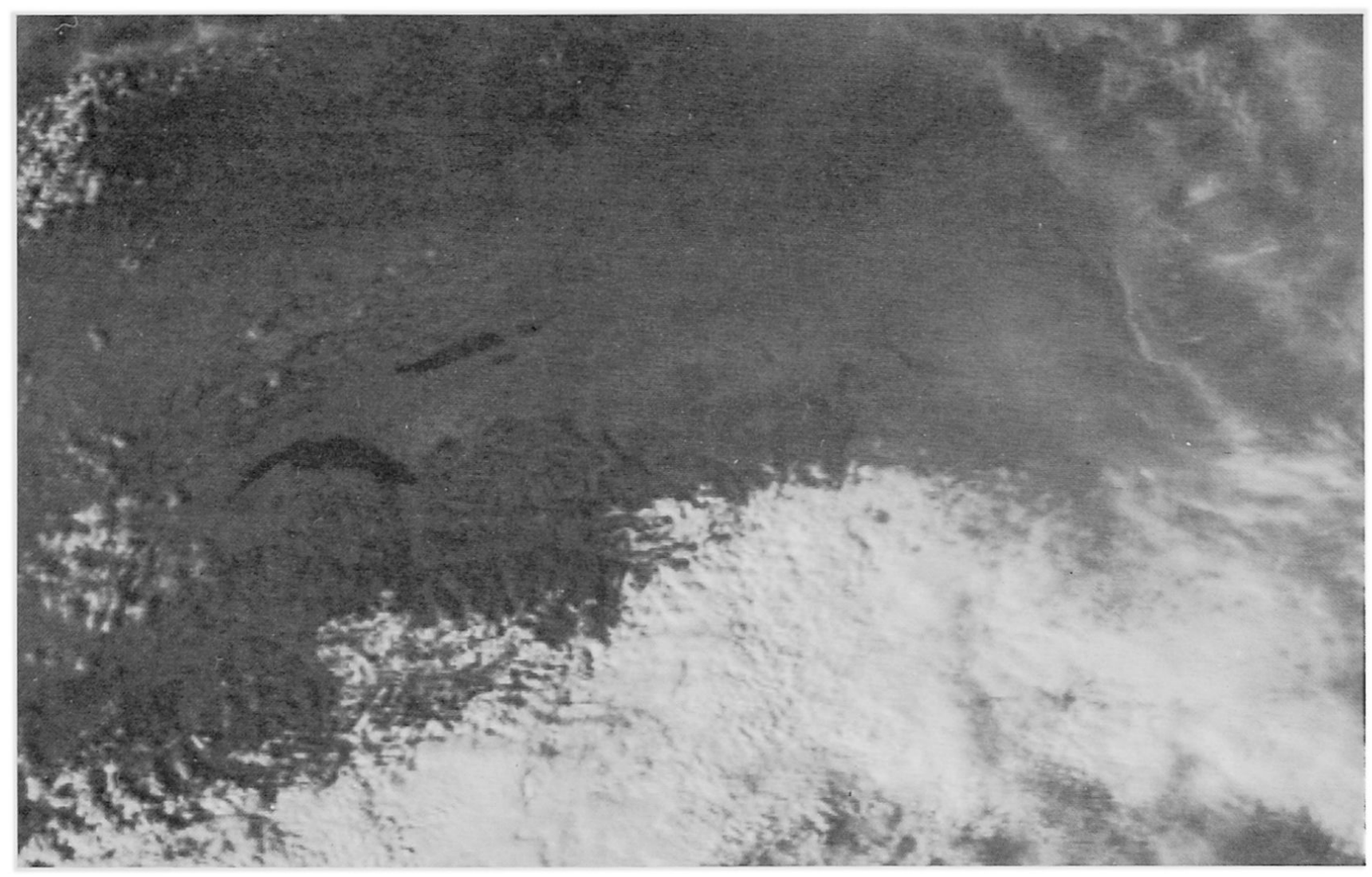

Figur 2 Satellitenbild von NOAA-7 vom 25. September 1982, 13.17UT (Südlage)

Als Folge der unterschiedlichen Blickrichtung und räumlichen Auflösung eignen sich Meteosat-2 und NOAA-Bilder unterschiedlich gut für die Kartierung der Bewölkung im Raume der Schweiz.

- Meteosat: Aufgrund der Geländeformen wird eine kleinräumige Differenzierung der Bewölkung erwartet. Die Pixel mit einer Größe von $2,5 \times 4 \mathrm{~km}^{2}$ können solche Verhältnisse nur rudimentär wiedergeben. Deshalb wurde in dieser Studie auf Meteosat-Bilder verzichtet. Das Wolkenfeld, das in B1 der Figur 1 vom Bodenbeobachter mit einem Achtel aufgezeichnet wird und unter den bereits erwähnten Bedingungen $6,6 \mathrm{~km}^{2}$ einnimmt, würde im Satellitenbild nicht als Einzelwolke in Erscheinung treten.

- NOAA-6,7 und 8: Sie liefern Aufnahmen in genügender räumlicher Auflösung (siehe Figur 2). Die Bildauswertung wird aber hauptsächlich erschwert durch die Verzerrung, die von Bild zu Bild ändert, und die schlechte Unterscheidbarkeit von Wolken und Schnee, auch wenn Bilder von mehreren Kanälen beigezogen werden.
Obwohl seit über 20 Jahren Wettersatelliten betrieben werden, sind die Zeitreihen lückenhaft und die Bilder von unterschiedlicher Qualität. Für die Kartierung der Bewölkungsverhältnisse in der Schweiz konnten 154 Satellitenbilder von NOAA-6,7 und 8 aus den Jahren 1982 und 1983 bearbeitet werden. Auf der Basis dieses kleinen Kollektives lassen sich nur eingeschränkt klimatologische Aussagen machen.

Zusammenfassend zeichnen sich terrestrische Bewölkungsbeobachtungen durch lange, homogene Beobachtungsreihen aus, Satellitenbeobachtungen durch räumlich homogene, flächendeckende Darstellungen der Bewölkungsverhältnisse. Als Nachteile stehen bei der einen Methode die punktuelle, räumlich heterogene Betrachtungsweise, bei der anderen die kurzen, lückenhaften Zeitreihen im Vordergrund.

Mit diesem Ausgangsmaterial soll nun versucht werden, eine Karte der mittleren Bewölkung für die Schweiz zu erstellen. Die klimatologischen Eckdaten sind dabei die mittleren Bewölkungswerte für die Jahre 1931 bis 1960 . Die flächenhaft differenzierende Information wird von den Satellitenbildern beigesteuert. 


\section{Die Bewölkungskarte}

\subsection{Einzelbildauswertung}

Satellitenbilder von NOAA- 6,7 und 8 sind in der Form, wie sie auf der Bodenstation empfangen werden, für unsere Zwecke noch nicht unmittelbar nutzbar. In einem ersten Verarbeitungsschritt geht es deshalb darum, die Bilder zu entzerren und die Wolken vom Hintergrund zu separieren. Für eine derartige Aufbereitung der Satellitendaten wurden automatische Verfahren entwickelt, die aber teure Bildverarbeitungssysteme voraussetzen. Weil das GIUB nicht über solche Installationen verfügt, mußte ein einfaches, visuelles Auswertungsverfahren gewählt werden. Es bestand in einer optischen Entzerrung am Zoom-Transferscoop und einer Schätzung der Bewölkung für Einheitsflächen, die dem Satellitenbild am selben Gerät und im selben Arbeitsschritt überblendet werden konnten (Figur 3, Arbeitsschritt 1).

Für die Flächenschätzung war deshalb die Schweiz zuvor in 173 topographische Flächen gegliedert worden, die sich in ihrer Bewölkung deutlich unterscheiden.

Die Bewölkung jeder Einheitsfläche wurde einer der Klassen $0-10 \%, 10-25 \%, 25-50 \%, 50-75 \%, 75-90 \%$ und 90-100\% Bedeckung zugeordnet (Figur 3, Arbeitsschritt 2). Die Wahl der Klassenbreiten basiert auf Erfahrungen, die mit Flächenschätzungen von Test«Personen» gesammelt wurden. Es hat sich nämlich gezeigt, daß Flächen mit einer Bedeckung um 5\% bzw. $95 \%$ exakter beurteilt werden als Flächen mit einer Bedeckung um $50 \%$. Diesem Schätzverhalten kann damit Rechnung getragen werden, daß im Bereich von $50 \%$ Bedeckung größere und um 5\% und 95\% Bedekkung kleinere Klassenbreiten gewählt werden. Die Gefahr einer falschen Zuordnung wird damit reduziert.

Um die Weiterverarbeitung mit dem Computer zu ermöglichen, wurden die 173 Bewölkungsschätzwerte jedes Satellitenbildes in eine Schweizerkarte mit $2 \times 2 \mathrm{~km}^{2}$ Raster umgerechnet und auf Disk gespeichert (Figur 3, Arbeitsschritte 3 und 4).

\subsection{Berechnung der Bewölkungskarte aus Bodenbeob- achtungen und Satellitenbildern}

\subsubsection{Lösungsmöglichkeiten}

Die Bewölkung ist räumlich und zeitlich starken Änderungen unterworfen. Die 154 ausgewerteten Satellitenaufnahmen sind deshalb nur Momentaufnahmen eines dynamischen Prozesses. Obwohl die Bilder einen zufälligen Zustand der Bewölkung festhalten, scheint die Bewölkungsverteilung trotzdem gewissen Regeln zu gehorchen:

- Es bildet sich beispielsweise im Winter bei Hochdrucklage häufig Nebel im Mittelland.
- Bei Nordstau ist die Alpensüdseite in der Regel wolkenfrei.

Die Hypothese ist deshalb naheliegend, daß die Bewölkungsverteilung abhängig ist von der Wetterlage. Mit Satellitenbildern lassen sich Karten mittlerer Bewölkung für jede Wetterlage berechnen, indem man genügend Bilder jeder Lage heraussucht, sie dann auf dem Computer addiert und daraus schließlich eine mittlere Bewölkungsverteilung berechnet. Diesem Prinzip folgend, wurden die 154, durch die Einzelbildauswertung bereits vorbereiteten Satellitenbilder verschiedenen Wetterlagen zugeordnet und zu Wetterlagenkarten weiterverarbeitet.

Zwei Wege sind nun denkbar, um über die Wetterlagenkarten zum Ziel, einer Karte der mittleren Bewölkung, zu gelangen:

1. Jede Wetterlagenkarte kann entsprechend der Häufigkeit, mit der die Wetterlage im langjährigen Mittel auftritt, gewichtet werden. Durch eine Addition der gewichteten Karten ist es möglich, die angestrebte Karte zu berechnen.

2. Das zweite Verfahren ähnelt in wesentlichen Aspekten dem bereits vorgestellten: Wiederum werden die einzelnen Wetterlagenkarten mit einem Faktor gewichtet, und wiederum werden die gewichteten Karten durch Addition aufeinandergeschichtet. Im Unterschied zum bereits vorgestellten Vorgehen entsprechen nun die Gewichtungsfaktoren nicht mehr zwingend den klimatologischen Häufigkeiten der Wetterlagen, sondern einem Zahlenwert, der in einem statistischen Verfahren ermittelt wird. Folgende Bedingung wird jetzt an die Gewichtungsfaktoren gestellt: Die aus der Gewichtung schließlich resultierende Karte der mittleren Bewölkung muß überall dort, wo Bodenstationen mit langjährigen Beobachtungsreihen lokalisiert sind, mit diesem Stationswert möglichst genau übereinstimmen. Liegen die Stationswerte und die berechneten Werte auf der Bewölkungskarte nahe beieinander, so kann angenommen werden, daß die Gewichtung der Wetterlagenkarten richtig ist und die berechnete Bewölkungskarte die Bewölkungsverteilung gut wiedergibt.

Die Gewichtungsfaktoren können mit Hilfe einer multiplen, linearen Regression gefunden werden.

Lösungsweg 1 ist wohl einfacher als das zweite Verfahren, ließ sich aber in unserem Falle nicht begehen, weil wir Wetterlagenkarten bestimmten, für die keine Häufigkeiten im langjährigen Mittel bekannt sind. Unser Vorgehen soll nun im nächsten Kapitel dargelegt werden.

\subsubsection{Der Regressionsansatz}

Ausgangsmaterial sind die 154 Satellitenaufnahmen, die in der Einzelbildauswertung vorverarbeitet wurden. Die Bilder wurden nun acht Wetterlagen, die mit 


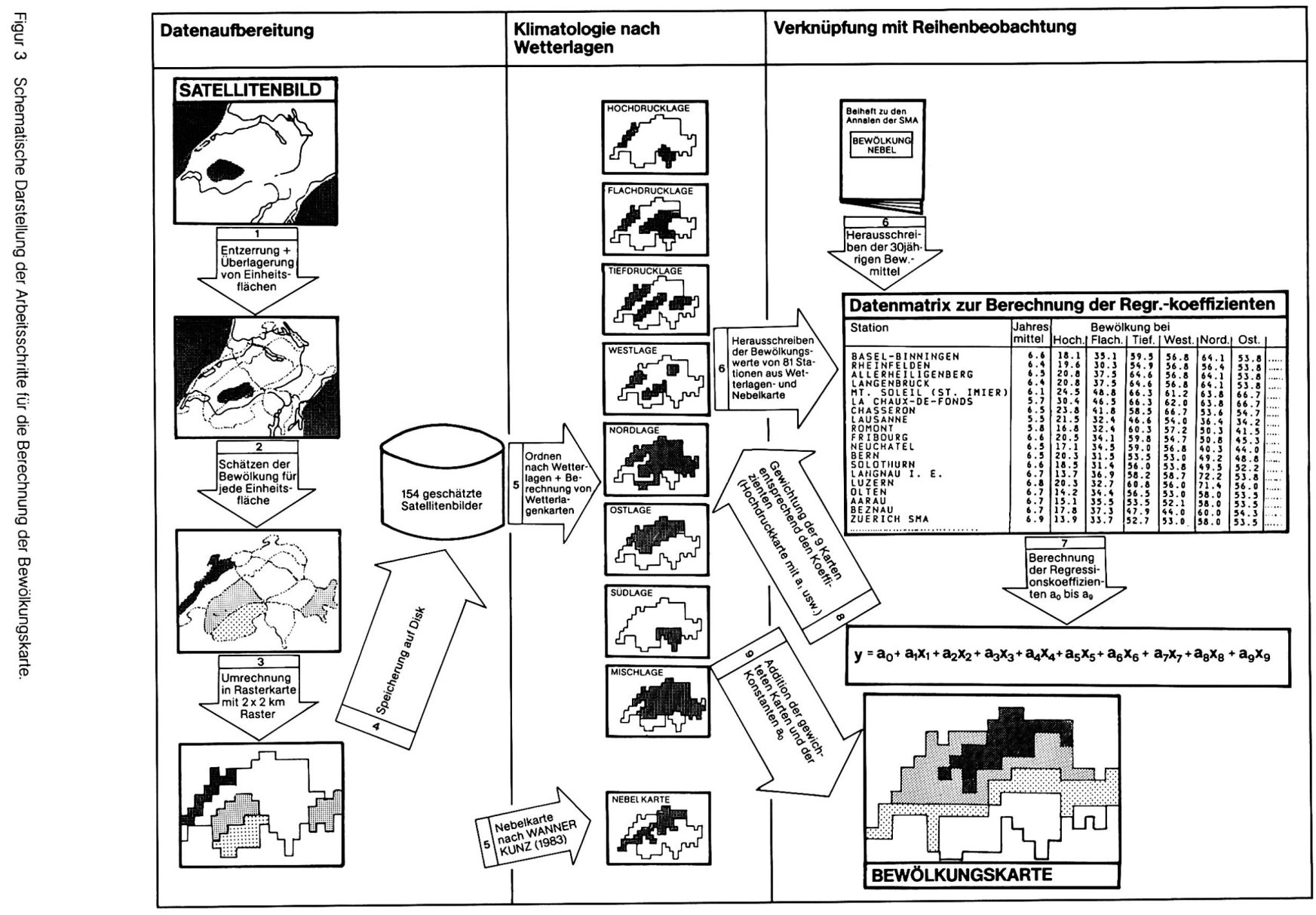



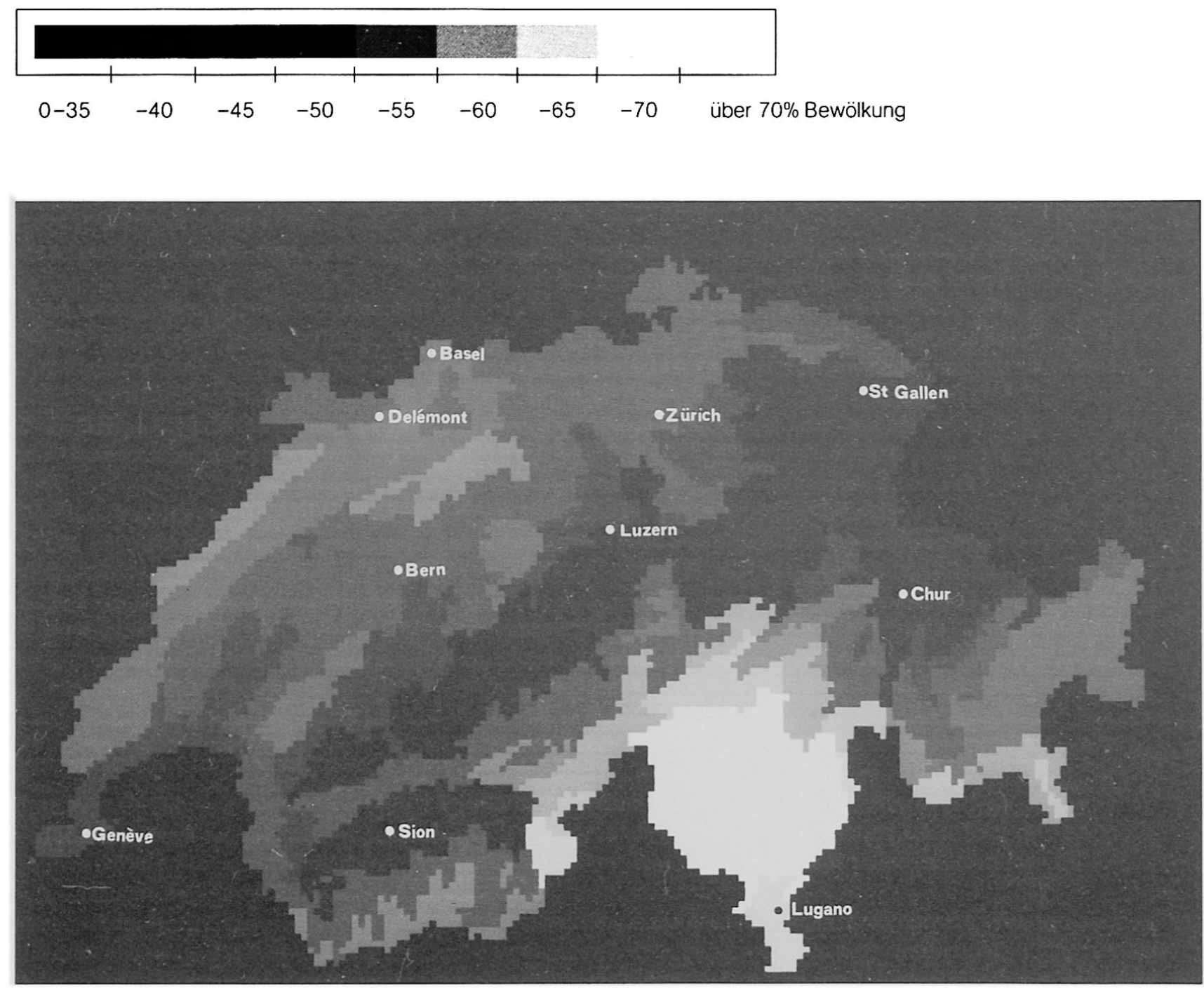

Figur 4 Bewölkungsverteilung bei Südlagen, berechnet mit 15 Satellitenbildern

Hilfe des Wetterlagenkalenders von SCHÜEPP (1968) definiert wurden, zugeordnet. Zur Berechnung der mittleren Bewölkung für jede Wetterlage standen minimal 15 (für Südlagen) und maximal 38 Satellitenbilder zur Verfügung (Flachdrucklagen). In Figur 4 ist die Wetterlagenkarte für Südlagen dargestellt.

Das Kollektiv der 154 Satellitenbilder enthält keine Nebelbilder. Konsequenz davon ist, da $\beta$ in den 8 Wetterlagenkarten die Information Nebel, die vor allem für Hochdrucklagen eine große Rolle spielt, ganz fehlt. Aus diesem Grund wurden die acht Wetterlagenkarten ergänzt durch eine 9. Karte, eine Nebelkarte der Schweiz. Es bot sich die Karte von WANNER/KUNZ (1983) an, die, wie die anderen Karten, in einem $2 \times 2-\mathrm{km}^{2}$-Raster bereits computergerecht vorlag und ebenfalls mit Satellitenbildern erstellt wurde.
Dem Umstand, daß die 8 Wetterlagenkarten keinen Nebel enthalten, ist es zuzuschreiben, daß nicht das erste Verfahren aus dem vorherigen Kapitel zur Anwendung kommen konnte.

In einem nächsten Arbeitsschritt soll nun nach den Faktoren gesucht werden, mit denen die 9 Karten gewichtet werden müssen, bevor dann alle zu einer Karte der mittleren Bewölkung addiert werden. Für die Berechnung der 9 Faktoren (Koeffizienten) interessieren vorerst von jeder Karte nur 81 Rasterflächen; nämlich diejenigen Flächen, in denen Stationen mit langjährigen Bewölkungsbeobachtungen liegen. Die Bewölkungswerte dieser Rasterflächen werden zu einer Tabelle zusammengestellt, die für jede Bodenstation die 9 Bewölkungswerte für 8 Wetterlagen und Nebel enthält. Die Datenmatrix wird ergänzt mit den Bewölkungsmittelwerten der 81 Stationen für die 
Jahre 1931-1960, die in einer Publikation von SCHÜEPP (1963) vorliegen (Figur 3, Arbeitsschritt 6). Gemäß unserer Grundidee soll nun abgeklärt werden, ob sich Gewichtungsfaktoren $a_{1}$ bis ag für die 9 Wetterlagen finden lassen, die angewendet auf die 9 Bewölkungswerte einer Bodenstation deren langjähriges Mittel möglichst genau ergeben. Mathematisch geht es darum, in der folgenden Formel die Koeffizienten $\mathrm{a}_{0}$ bis ag zu finden:

$y=a_{0}+a_{1} x_{1}+a_{2} x_{2}+a_{3} x_{3}+a_{4} x_{4}+a_{5} x_{5}$ $+a_{6} x_{6}+a_{7} x_{7}+a_{8} x_{8}+a_{9} x_{9}$

- abhängige Variable

y: Jahresmittel der Bewölkung

- unabhängige Variable

$\mathrm{x}_{1}$ : Bewölkung bei Hochdrucklagen, entnommen aus der Bewölkungskarte für Hochdrucklagen

$\mathrm{x}_{2}$ : Bewölkung bei Flachdrucklagen

$\mathrm{x}_{3}$ : Bewölkung bei Tiefdrucklagen bis...

$\mathrm{x}_{9}$ : Bedeckung bei Nebel

- Konstante $a_{0}$

- Koeffizienten

$a_{1}$ : Gewichtungsfaktor für Hochdrucklage

$\mathrm{a}_{2}$ : Gewichtungsfaktor für Flachdrucklage

bis...

ag: Gewichtungsfaktor der Nebelkarte

Mit Hilfe der Datenmatrix, die oben erstellt wurde, können die Koeffizienten einfach ermittelt werden (Figur 3, Arbeitsschritt 7). Die Qualität dieser Werte $\mathrm{a}_{0}$ bis ag läßt sich überprüfen, indem man sie auf die Bewölkungswerte $x_{1}$ bis $x_{9}$ der 81 Bodenstationen entsprechend obiger Gleichung anwendet und die daraus resultierenden Bewölkungswerte mit den 81 Bodenbeobachtungen vergleicht. Diese Überprüfung hat ergeben, daß die berechneten Werte von den beobachteten im Mittel um 2,8\% Bedeckung (= Standardabweichung) abweichen und eine Korrelation von 0,89 zwischen den beiden Werten besteht. Die hohe Korrelation und kleine Standardabweichung, die etwa $10 \%$ der Variationsbreite der Bewölkung in der Schweiz ausmacht, lassen den Schluß zu, daß mit Hilfe der gefundenen Koeffizienten die langjährigen Bewölkungsmittelwerte gut berechnet werden können. Damit werden wir von den Bewölkungswerten der Bodenstationen unabhängig und in die Lage versetzt, über die Gewichtung und Addition der neun Bewölkungskarten die mittlere Bewölkung für die Schweiz zu berechnen (Figur 3, Arbeitsschritte 8 und 9). Wir verfügen über ein Modell zur Berechnung der mittleren Bewölkung in der Schweiz.

Die nach dem beschriebenen Verfahren berechnete Bewölkungskarte soll im nächsten Kapitel besprochen werden.

\section{Interpretation und Kritik der Bewölkungskarte}

\subsection{Vergleich mit der Bewölkungskarte von Schuepp}

Die berechnete Bewölkungskarte soll anhand eines Vergleichs mit der Bewölkungskarte von SCHÜEPP (1978) vorgestellt werden (Figur 5).

Beiden Karten gemeinsam ist das Zeitintervall, für das sie die mittlere Bewölkung wiedergeben: die Periode 1931-1960. Sie stimmen auch in der bewölkungsklimatologischen Grobgliederung der Schweiz überein:

1. Mittelland und östliche Teile des Juras als Gebiete mit den höchsten, mittleren Bewölkungswerten.

2. Alpensüdseite (Wallis, Tessin, Engadin, Münstertal, Puschlav und Bergell) mit geringster Bewölkung im Raume der Schweiz.

3. Westlicher Jura, Genferseegebiet, Voralpen und Alpen mit einer mittleren Bewölkung von 55 bis $65 \%$ Bedeckung.

In der Wiedergabe von Details unterscheiden sich die beiden Karten deutlich. In unserer Karte treten das Mittelland und der östliche Jura als reich gegliederte Regionen in Erscheinung. Deutlich zeichnen sich das untere Thurtal und die Täler des Luzerner Mittellandes ab, die unter einem Niveau von $700 \mathrm{~m}$ liegen, einer häufig beobachteten Nebelgrenze, und deshalb höchste Bewölkungswerte aufweisen. Dieselben Gründe liegen auch für die hohe Bewölkung in der Achse Jegenstorf - Koppigen - Aarwangen vor. $\mathrm{Da}$ auch im Jura der Nebel von lokaler Bedeutung sein kann, belegen die hohen Werte im Delsbergerbecken und im Laufental.

Obwohl auch in der Westschweiz der Jurasüdfuß hohe Bewölkungswerte aufweist, scheint doch hier die Romandie gegenüber der Deutschschweiz gewisse Vorteile zu genießen. Sie lassen sich damit erklären, daß sich insbesondere bei Nordlagen Föhneffekte (Joran) im Lee des westlichen Juras einstellen, die für die Auflösung der Wolken in höheren Niveaus verantwortlich sind.

Markante Differenzen bestehen zwischen den beiden Karten in der Beurteilung des Aaretales zwischen Bern und Thun. Während in der SCHUEPP-Karte die Zone höchster Bewölkung bei Bern eine Grenze findet, fingert sie in unserer Karte bis in die Region des Thunersees aus, was aufgrund der Nebeluntersuchungen von WANNER (1979) der Realität gut entsprechen dürfte.

In der Rangliste der Regionen mit geringster Bewölkung liegt in unserer Bewölkungskarte das Tessin vor dem Wallis und dem Engadin mit seinen südlich angrenzenden Tälern. Ob die Verhältnisse damit aber richtig wiedergegeben werden, kann hier nicht beurteilt werden, denn diese Rangfolge findet sich beispielsweise in der Bewölkungskarte von SCHÜEPP nicht. 

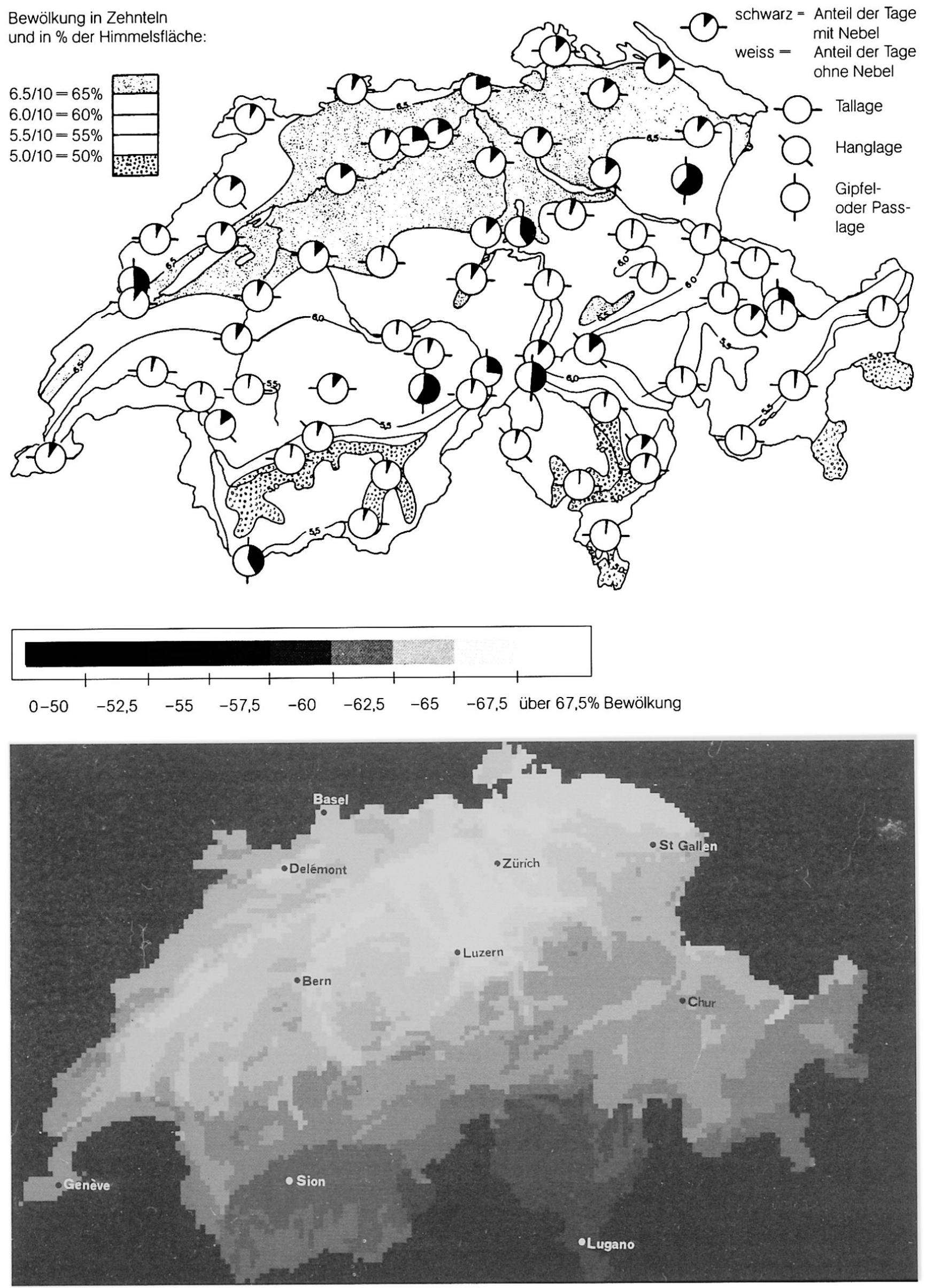

Figur 5 Vergleich der Bewölkungskarten 1931-1960 von SCHÜEPP (1978) und berechnet mit Regressionsmodell (unten). 
Auf ein Detail im Kanton Wallis möchten wir noch hinweisen. Die Region südlich des Simplon-Passes unterscheidet sich vom übrigen Kantonsteil durch deutlich geringere, mittlere Bewölkung und gehört damit bewölkungsklimatologisch eher zum Tessin.

In den übrigen Regionen überlagern sich Hochnebel, Staubewölkung und Lee-Effekte und bewirken große Bewölkungsunterschiede auf kurze Horizontaldistanzen. Für das Val de Travers und Vallée de Joux lassen sich die relativ geringen Bewölkungswerte mit der Wolkenauflösung im Lee der nordwestlich vorgelagerten Gebirgszüge erklären, dieselbe Erklärung dürfte auch auf das Tal der Le Javro (Valsainte), den Talkessel von Grindelwald, die Achse Maderaner-, Reussund Urserental und das Vorderrheintal zutreffen.

Bemerkenswert auch, daß sich durch unsere Berechnungen in den westlichen Hochalpen tiefere Bewölkungswerte ergeben als in den östlichen Gipfelregionen.

$\mathrm{Ob}$ sich dahinter eine klimatologische Tatsache verbirgt, kann noch nicht beurteilt werden.

\subsection{Schlussbemerkung}

Der abschließende Vergleich hat gezeigt, daß es mit der vorgestellten Methode möglich ist, eine Karte zu berechnen, welche die Bewölkungsverhältnisse detailliert wiederzugeben vermag. In diesem Sinne leistet sie einen Beitrag zum besseren Verständnis der Bewölkung in der Schweiz.

Zusätzlich zeigt das gewählte Verfahren einen Weg auf, um Boden- und Satellitendaten, eine punktuelle und eine flächenhafte Beobachtungsmethode, eine langjährige Meßreihe und wenige (154) Stichproben sinnvoll miteinander zu verknüpfen.

Die Methode weist aber auch Schwachstellen auf, die kurz aufgelistet werden sollen:

- Wir haben bei der Beschreibung des Datenmaterials darauf hingewiesen, daß Bodenstationen im allgemeinen die Bewölkung überschätzen. Damit fallen auch die langjährigen Bewölkungsmittelwerte, die wir als Grundlage zur Berechnung der Gewichtungsfaktoren benützen, zu hoch aus. Diese systematischen Schätzfehler übernehmen wir mit unserer Methode auch in die Bewölkungskarte. Die "wirklichen Bewölkungswerte» dürften aufgrund von Untersuchungen verschiedener Autoren und eigenen Abklärungen durchschnittlich um 5-10\% unter den beobachteten Werten der Bodenstationen liegen.

- Mit der Schätzung der Bewölkung für Einheitsflächen mit einer durchschnittlichen Größe des Kantons Zug konnte der Detailreichtum der Satellitenbilder nur ungenügend genutzt werden.

- Die Gliederung des Bildmaterials nach dem Wetterlagenkalender von SCHÜEPP konnte nicht restlos überzeugen und sollte eventuell durch eine andere Klassierung ersetzt werden.
- Das Kollektiv der 154 Satellitenbilder besteht zu drei Vierteln aus NOAA-7 - Nachmittagsaufnahmen. Mit dem vermehrten Einbezug von Morgenaufnahmen könnten die Wetterlagenkarten und damit auch die Bewölkungskarte verbessert werden (Berücksichtigung des Tagesganges).

\section{Dank}

Ganz herzlich bedanke ich mich bei Dr. M. Winiger und Dr. S. Kunz, die mich mit Anregungen und Kritik während der Arbeit begleitet haben. Besten Dank auch dem Geographischen Institut der Universität Bern, dem Schweiz. Nationalfonds zur Förderung der wissenschaftlichen Forschung und dem Eidgenössischen Institut für Reaktorforschung, die die Satellitenempfangsanlage immer wieder großzügig unterstützt haben.

\section{Abstract}

In this paper a map of cloud cover of Switzerland is presented, which is based on satellite data and the observations made by meteorological ground stations. First, the way the spatial information of meteorological satellites (covering 1 year) combined with the observations of ground stations during a period of 30 years, and how this was done with the aid of a regressions model, is explained. With this method, efficient use of the combination of satellite and ground data was attempted.

Secondly, the new cloud map of Switzerland is discussed and then compared with the cloud map made by SCHUEPP (1978).

\section{Literatur}

HEEB, M; 1983: Kartierung der Bewölkung mit Satellitenbildern. Diplomarbeit GIUB.

KUNZ, S.; VOLZ, R.; WITMER, U.; 1984: Sonnenenergienutzungszonen Schweiz - SONUS. Ein Kataster mit Strahlungsdaten für die Sonnenenergienutzung für die Gemeinden der Schweiz. GIUB.

SCHÜEPP, M., 1963: Bewölkung und Nebel. Beiheft z. d. Ann. d. Sz. Met. Anstalt. Zürich.

SCHÜEPP, M., 1968: Kalender der Wetter- und Witterungslagen von 1955 bis 1967 im zentralen Alpengebiet. Veröffentlichung d. Sz. Met. Anstalt, Nr. 11.

SCHÜEPP, M. et al., 1978: Regionale Klimabeschreibung, 1. Teil: Gesamtübersicht, Westschweiz, Wallis, Jura und Juranordfuß sowie Mittelland. Beiheft z. d. Ann. d. Sz. Met. Anstalt. Zürich.

WANNER, H., 1979: Zur Bildung, Verteilung und Vorhersage winterlicher Nebel im Querschnitt Jura - Alpen. Geographica Bernensia, Band G7.

WANNER, H.; KUNZ, S., 1983: Klimatologie der Nebel- und Kaltluftkörper im Schweizerischen Alpenvorland mit Hilfe von Wettersatellitenbildern. Arch. Met. Beoph. Biocl., Ser. B, $33: 31-56$. 